\title{
Molecular analysis of Dichelobacter nodosus isolated from footrot in sheep in Malaysia
}

\begin{abstract}
Pulsed field gel electrophoresis analysis of genomic DNA was used to investigate genetic diversity among Dichelobacter nodosus from footrot in sheep in Malaysia. Twelve Dichelobacter nodosus strains isolated from lesion materials from infected sheep were confirmed as Dichelobacter nodosus by polymerase chain reaction technique using the species-specific Dichelobacter nodosus 16S RNA sequence Ac and C as primers. Pulsed field gel electrophoresis banding profiles using restriction enzymes ApaI (5 HGGCCC3 Nj SfiI (5)GCCNNNNNGGCC3) Nind SmaI ( 1 CCCGGG3Njenabled the 12 Dichelobacter nodosus strains to be differentiated into eight different PFGE patterns and thus genome-types, with $\mathrm{F}$ (coefficient of similarity) values ranging from 0.17 to 1.0 (ApaI), 0.14 to 1.0 (SfiI) and 0.22 to 1.0 (SmaI). Strains with origin in different farms were shown to have different PFGE patterns (two strains, M7 and M8 were the only exception). On the basis of their PFGE, all field strains used in the study differed from the reference strains. Our data revealed that there are several clonal types of Dichelobacter nodosus isolates and indicated that there is probably more than one source of this pathogen on the farms studied. The study showed that strains of D. nodosus exhibited considerable genetic diversity using this method and that genomic analysis by pulsed field gel electrophoresis was useful in discriminating the D. nodosus strains.
\end{abstract}

Keyword: Dichelobacter nodosus; Sheep-bacteria; PCR; PFGE 